\title{
INFLUENCE OF THIN SURFACE LAYER ON FISSION GAS RELEASE FROM URANIUM DIOXIDE SINGLE CRYSTAL DURING IRRADIATION
}

\author{
M. SzUTA \\ Institute of Atomic Energy, 05-400 Otwock-Świerk, Poland
}

\begin{abstract}
In the present paper it is assumed that in the fluence range of $4 \times 10^{19}-0.5 \times 10^{21}$ fissions $/ \mathrm{cm}^{3}$ the main contribution to the fission gas release from an uranium dioxide single crystal is from the bubble traps by knock-out process. An analysis of bubble distribution in the single crystal for different temperatures shows that some experimental peculiarities of the fission gas release during irradiation arise from different conditions of the thin surface layer and the interior of the considered solid. The thin surface layer is assigned by the fission fragment range.
\end{abstract}

PACS numbers: 25.85.Ec, 66.30.Lw

\section{Introduction}

Analysis of fission gas release from $\mathrm{UO}_{2}$ during irradiation gives evidence that the surface of a solid presents conditions different from those of the interior. Thus it is sometimes doubtful whether gas-release measurements reflect the volume characteristics of the solid or the surface characteristics. This is clearly seen for the experiments above the fission fluence equal to $4 \times 10^{19}$ fissions $/ \mathrm{cm}^{3}$ when bubbles are formed.

In the fission fluence range of $4 \times 10^{19}-0.5 \times 10^{21}$ fissions $/ \mathrm{cm}^{3}$ the main contribution to the fission gas release from the $\mathrm{UO}_{2}$ single crystal is from the bubble traps by knock-out process and diffusion of bubbles [1-3].

Most experiments of fission gas release pertain to the above mentioned range. Many unexplained anomalies exist within the experimental results presented in the open literature. Of such anomalies we could mention the following:

a) Fission gas release is caused by a combination of two basic processes: a temperature independent process and a temperature dependent process [4].

b) Fission gas release is dependent on a decay constant and the fission gas has the same proportions of isotopes for all temperatures, both in the temperature dependent region and the non-temperature dependent region [4]. 
c) Contrary to expectations of the classical diffusion, the tendency for xenon to migrate as fast as krypton is noted [5].

The anomalies (a) and (b) suggest that the same transport mechanism brings the gas to the specimen surface, even though the release is by two different processes: the temperature independent process and the temperature dependent process. The recoil process is assumed to be the transport mechanism to the specinen surface. The recoil process which controls the fission gas product release from the material affects the $\mathrm{UO}_{2}$ fuel surface layer to a depth not more than $10 \mu \mathrm{m}$ - the fission fragment range.

The fact of occurrence of the same ratio of the released gas isotopes for both the temperature dependent and temperature independent region and the fact that xenon migrates as fast as krypton implies that the same carriers of fission gas operate in both temperature regions.

As it is known [6-8] that the fission gas products can be in three different states (gas in bubbles, soluted gas, diffusing gas) the carriers of fission gas accomplishing the above experimental data can only be the bubbles.

Therefore it is obvious that better understanding of influence of thin surface layer on fission gas release in the fluence range of $4 \times 10^{19}-0.5 \times 10^{21}$ fissions $/ \mathrm{cm}^{3}$ can be obtained by analysis of the gas bubble distribution in the $\mathrm{UO}_{2}$ single crystal.

\section{Bubble distribution in a single crystal of uranium dioxide during irradiation}

It is experimentally proved that the formed gas bubbles are destroyed by the fission fragments [6]. In the thin surface layer the bubble elimination rate can be classified into two categories: (a) The rate in which the fission fragment track does not cross the surface layer of the crystal and the kinetically excited gas atoms of the bubble become knocked back into the matrix - this process is termed as an irradiation induced solution. Gas in this state is immobile. (b) The rate in which the fission track does cross the surface and the kinetically excited gas atoms of the bubble follow the fission fragment out of the bubble - this process is termed here as knock-out release process.

In the bulk of the $\mathrm{UO}_{2}$ single crystal the bubble elimination is done only by the irradiation induced solution.

The trouble is that the analysis of the behaviour of the gas bubble during irradiation cannot be limited exclusively to the bubbles.

It is assumed that the existing gas in the $\mathrm{UO}_{2}$ crystal which occurs in three extreme states (gas trapped in the bubbles, diffusing gas and gas soluted in the crystal) have to occur in an intermediate state during irradiation as well. In the stable state during irradiation, the intermediate gas after thermalization can be trapped by the bubble, soluted in the material or become diffusing.

Along the fragment trajectories in $\mathrm{UO}_{2}$ a secondary strong thermal spikes occur. Gas atoms caught up in these spikes (kinetically excited) together with gas fragments are free to migrate as random walkers. Consequently, the bubble migration is an entangled process of the following sequence "kinetic excitation of gas atoms, intermediate gas formation and bubble formation at their new location". 
A bubble can be eliminated if a fission fragment passes in the direct vicinity of the bubble or directly through it. This means that the rate of bubble elimination is directly proportional to the product of fission rate and bubble concentration.

During irradiation the bubbles can diffuse at large distances towards the surface, but they do not reach the surface because of knock-out bubble elimination.

Since the probability of bubble elimination by knock-out is remarkably higher than the bubble elimination by irradiation induced solution, therefore the bubble concentration in the surface layer of thickness not higher than the fission fragment range is far less than in the bulk of the specimen.

The concentration of bubbles in the surface layer consists of the average concentration of the created bubbles in the surface layer and the concentration of the diffused bubbles into the surface layer. In order to solve the problem of bubble distribution in the $\mathrm{UO}_{2}$ single crystal, it is assumed to write separate equations for the concentration of bubbles created in the surface layer $\left(N_{\mathrm{tr}}^{\mathrm{ko}}\right)$ and these bubbles which diffused from the bulk of the $\mathrm{UO}_{2}$ crystal $\left(N_{\mathrm{trI}}^{\mathrm{D}}(x)\right)$ and concentration of bubbles in the bulk of the crystal $\left(N_{\mathrm{trII}}^{\mathrm{D}}(x)\right)$ :

$$
\begin{aligned}
& \frac{\mathrm{d} N_{\mathrm{tr}}^{\mathrm{ko}}}{\mathrm{d} t}=g_{1} f-\left(g_{2}+g_{3}\right) f N_{\mathrm{tr}}^{\mathrm{ko}} \quad \text { for } 0 \leq x \leq r, \\
& \frac{\partial N_{\mathrm{trI}}^{\mathrm{D}}}{\partial t}=D_{\mathrm{b}} \nabla^{2} N_{\mathrm{trI}}^{\mathrm{D}}-\left(g_{2}+g_{3}\right) f N_{\mathrm{trI}}^{\mathrm{D}} \quad \text { for } \quad 0 \leq x \leq r, \\
& \frac{\partial N_{\mathrm{trII}}^{\mathrm{D}}}{\partial t}=D_{\mathrm{b}} \nabla^{2} N_{\mathrm{trII}}^{\mathrm{D}}+g_{1} f-g_{3} f N_{\mathrm{trII}}^{\mathrm{D}} \text { for } \quad r \leq x<\infty,
\end{aligned}
$$

where $f-$ fission rate, $t$ - time, $r$ - fission product range, $x-$ distance into the fuel from the sample surface, $D_{\mathrm{b}}-$ bubble diffusion coefficient, $D_{\mathrm{b}}=$ $D_{0 \mathrm{~b}} \exp (-Q / R T), D_{0 \mathrm{~b}}$ - pre-exponential constant, $R$ - universal gas constant, $T$ - absolute temperature, $g_{1}, g_{2}, g_{3}$ - constants.

Considering that for the stable state the rate of change of bubble concentration is equal to zero and further for the semispace there is only one dimension problem, Eqs. (1)-(3) were simplified. The solution of the simplified Eqs. (2) and (3) is done for the assumption of spatially uniform temperature and fission rate and for the following: boundary conditions of bubble concentration distribution $N_{\mathrm{trl}}^{\mathrm{D}}(x)=0$ at $x=0 ; N_{\mathrm{trII}}^{\mathrm{D}}(x)=g_{1} / g_{3}$ at $x=\infty$, the sewing up condition $N_{\mathrm{trl}}^{\mathrm{D}}(r)=N_{\mathrm{trII}}^{\mathrm{D}}(r)$ and the continuity condition that the first derivatives of the bubble concentration at $x=r$ are the same

$$
\begin{aligned}
& N_{\mathrm{trI}}^{\mathrm{D}}(x)=2 C_{1} \sinh \left(\beta_{1} x\right) \quad \text { for } \quad 0 \leq x \leq r, \\
& N_{\mathrm{trII}}^{\mathrm{D}}=C_{2} \exp \left(-\beta_{2} x\right)+\frac{g_{1}}{g_{3}} \text { for } \quad r \leq x \leq \infty,
\end{aligned}
$$

where

$$
\begin{aligned}
& \beta_{1}=\sqrt{\frac{\left(g_{2}+g_{3}\right) f}{D_{\mathrm{b}}}}, \quad \beta_{2}=\sqrt{\frac{g_{3} f}{D_{\mathrm{b}}}}, \\
& C_{1}=\frac{g_{1} / g_{2}}{\left[\exp \left(\beta_{1} r\right)-\exp \left(-\beta_{1} r\right)\right]+\left(\beta_{1} / \beta_{2}\right)\left[\exp \left(\beta_{1} r\right)+\exp \left(-\beta_{1} r\right)\right]}, \\
& C_{2}=-\frac{\left(g_{1} \beta_{1} / g_{3} \beta_{2}\right)\left[\exp \left(\beta_{1} r\right)+\exp \left(-\beta_{1} r\right)\right]}{\exp \left(-\beta_{2} r\right)\left\{\left[\exp \left(\beta_{1} r\right)-\exp \left(-\beta_{1} r\right)\right]+\left(\beta_{1} / \beta_{2}\right)\left[\exp \left(\beta_{1} r\right)+\exp \left(-\beta_{1} r\right)\right]\right\}} .
\end{aligned}
$$




\section{Discussion}

Figure 1 shows two curves (for constant temperatures $1138 \mathrm{~K}$ and $1613 \mathrm{~K}$ ) of the bubble concentration in function of distance from the surface of the semispace of $\mathrm{UO}_{2}$ single crystal for the constant fission rate $f=1.0 \times 10^{12}$ fissions $/\left(\mathrm{cm}^{3} \mathrm{~s}\right)$ and for the following constants: $g_{1}=10, g_{2}=10^{-15} \mathrm{~cm}^{3}, g_{3}=10^{-17} \mathrm{~cm}^{3}$, $D_{0 b}=4.6 \times 10^{-8} \mathrm{~cm}^{2} \mathrm{~s}^{-1}$ and $Q=1.0886 \times 10^{5} \mathrm{~J} / \mathrm{mol}$. Some comments on the estimation of the constant parameters were presented previously [1-3].

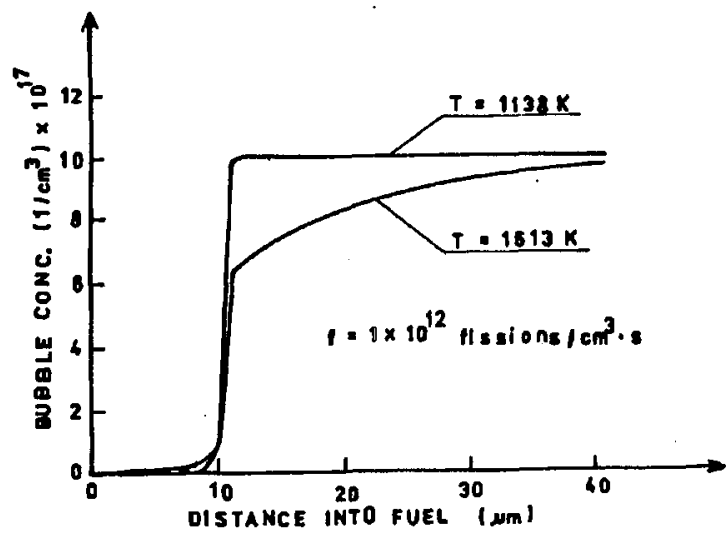

Fig. 1. Calculated concentration of bubbles in the surface layer of the uranium dioxide single crystal.

The higher concentration of bubbles for the lower temperature than for the higher temperature explains the burst of fission gas release when the temperature is increased [4]. Because these two curves present the distribution of bubbles for stable state, therefore during transient state the excess of the bubbles contained between these two curves appear in the release of fission gas products as a burst for a step function of the temperature. When the single crystal is decreased then there is no excess of the bubbles and it is obvious that the burst does not occur and rather a smooth transition to the lower release rate is observed.

This explains also (see Fig. 1) the observation of Matthews and Small [9] that zones depleted of bubbles are seen adjacent to the grain boundaries. The higher temperature i.e. the higher release rate, the bigger zone of low bubble concentration near the $\mathrm{UO}_{2}$ crystal surface is predicted.

When the specimen is maintained at a constant fission rate and the temperature is sinusoidally changed, we can anticipate that the maximum release rate will occur before the specimen reaches its maximum temperature, while the gas release rate and temperature will reach minimum value at the same time, because of the excess of bubbles when the temperature is increased. This effect was observed in the experiments of Carroll and Sisman [10].

On the basis of the above presented processes and the analysis of bubble distribution in the single crystal for two different temperatures, there is no doubt 
that some peculiarities of the fission gas release arise from different conditions of the surface and the interior of the considered solid.

It is worth noting that in addition to the above, the defect trap model explains simultaneously the peculiar observations of the fission gas release presented in the open literature.

\section{References}

[1] M. Szuta, J. Nucl. Mater. 58, 278 (1975).

[2] M. Szuta, J. Nucl. Mater. 130, 434 (1985).

[3] M. Szuta, J. Nucl. Mater. 210, 178 (1994).

[4] R.M. Carroll, O. Sisman, Nucl. Sci. Eng. 21, 147 (1965).

[5] J.A. Turnbull, R.J. White, C. Wise, in: Prac. IAEA Tech. Committee Mtg. in Water Reactor Fuel Element Computer Modelling in Steady State, Transient and Accident Conditions, Preston (England) 1988, IAEA, Vienna 1989, p. 174.

[6] J.A. Turnbull, J. Nucl. Mater. 38, 203 (1971).

[7] J.A. Turnbull, R.M. Cornell, J. Nucl. Mater. 41, 156 (1976).

[8] C. Syros, J. Sakellaridis, C. Ronchi, J. Nucl. Mater. 168, 65 (1989).

[9] J.R. Matthews, G.J. Small, in: Proc. IAEA Tech. Committee Mtg. on Water Reactor Fuel Element Computer Modelling in Steady State, Transient and Accident Conditions, Preston (England) 1988, IAEA, Vienna 1989, p. 195.

[10] R.M. Carroll, O. Sisman, Nucl. Appl. 2, 142 (1966). 